\title{
Physiological Demands, Morphological Characteristics, Physical Abilities and Injuries of Female Soccer Players
}

\author{
by \\ Zoran Milanović ${ }^{1}$, Goran Sporiš ${ }^{2}$, Nic James ${ }^{3}$, Nebojša Trajković, \\ Aleksandar Ignjatović, Hugo Sarmento ${ }^{6}$, Athos Trecroci ${ }^{7}$, \\ Bruno Miguel Borges Mendes ${ }^{8}$
}

The popularity of female soccer is increasing as well as the number of females playing soccer. Similarly, over the last twenty or so years, research in soccer has increased significantly, but a large disparity exists in the volume of studies involving male and female players. As a consequence of this, female players remain less well understood compared to males. The purpose of the present narrative review was to describe morphological characteristics, physiological demands, physical abilities and injuries in female soccer players. Physiological demands are similar between men's and women's soccer, but competitive women's matches were characterized by nearly 33\% less distance covered, although at higher intensity levels (maximum speeds greater than $15 \mathrm{~km} / \mathrm{h}$ ) than typically found in the men's game. Sub-elite female players also tended to run less at higher intensity levels at the end of both halves in comparison with elite female players. High intensity running is an important factor of success in soccer since many critical moments of the game occur under this condition. The ability to rapidly change direction also determined elite, sub-elite and amateur levels. The implementation of functional training, which focused on soccer-specific drills and plyometric exercises, to improve explosive power, may improve conditioning in female soccer players as well as decrease the risk of injuries which was 3-8 times higher in females compared to males. This review presents an in-depth overview of the most influential factors for determining success in female soccer.

Key words: women's soccer, match performance, female athletes.

\section{Introduction}

Female soccer has increased in popularity at all levels of play, with an estimated 6.3 million participants over 7 years of age (Olson, 2008). While research into soccer has significantly increased in the last twenty or so years, there still exists a large disparity in the volume of studies involving male and female players. Research that has had the most significant influence on soccer development investigated the physical demands, on-pitch technical and tactical performance, morphological characteristics of players, optimal training methods for preventing injuries, recovery

1 - Faculty of Sport and Physical Education, University of Nis, Nis, Serbia.

2 - Faculty of Kinesiology, University of Zagreb, Zagreb, Croatia.

3 - London Sport Institute, Faculty of Science and Technology, Middlesex University, London, UK.

4 - Faculty of Sport and Physical Education, University of Novi Sad, Novi Sad, Serbia.

5 - Faculty of Education, University of Kragujevac, Jagodina, Serbia.

6 - Research Unit for Sport and Physical Activity (CIDAF), Faculty of Sport Sciences and Physical Education, University of Coimbra, Coimbra, Portugal.

7 - Department of Biomedical Sciences for Health, Università degli studi di Milano, Milano, Italy.

8 - Head of F.C. Benfica LAB, Sport Lisboa e Benfica, Lisboa, Portugal. 
methods and conditioning drills. As a consequence of the limited research in female soccer, the female player remains less well understood than the male counterpart. This is not surprising however, since a) there has only been a two generation span of elite female participation in soccer; b) there are fewer females involved in playing and coaching; and c) research tends to converge on areas which can provide funding.

Sport and exercise scientists involved in soccer research have been investigating the multitude of factors that contribute to the performance of a player (Rey et al., 2012). For example, while most of the game is aerobic in nature, the most influential skills, typically observed in players in close proximity and relevant to the on ball activity, such as the ability to jump and sprint during challenges with opponents, are anaerobic (Bangsbo, 1993). These requirements will be very similar in the women's game, with high levels of aerobic capacity (Mohr et al., 2008), muscular strength and endurance (Grieco et al., 2012), speed, speed endurance, agility (Polman et al., 2004) and flexibility (Davis and Brewer, 1993) required. However a much cited global indicator of the physical demands of soccer is suggested to be the total distance covered by a player during a match. Some studies (Andersson et al., 2010; Krustrup et al., 2005; Mohr et al., 2008) have reported that female soccer players typically cover $9000-11000 \mathrm{~m}$ during a match. Furthermore, Gabbett and Mulvey (2008) suggest that the level of competition influences this value.

The purpose of the present narrative review was to describe morphological characteristics, physiological demands, physical abilities and injuries in female soccer players. As discussed below, all aforementioned components appear to evoke important fitness abilities which could discriminate elite, sub-elite and amateur levels. For that reason, all results were compared between top level, high level and amateur female soccer players. We hypothesized that better understanding of morphological characteristics, physiological and physical demands of female soccer would lead to better criteria for a future selection process and players development.

\section{Morphological characteristics}

Research into the influence of morphological characteristics on soccer performance is not straightforward, mainly because the individual characteristics of players and their playing positions confound the findings. Reilly et al. (1990) proposed that body composition played an important role in the fitness of male soccer players. However, caution is advisable when making direct comparisons between male and female players as males tend to have a higher ratio of lean body mass to body fat than female players ( $\mathrm{R}$ Matković et al., 2003) which may adversely affect the endurance of female players. Martens (1996) found that female professional soccer players tended to be mesomorphic whereas non-players were more endomorphic or ectomorphic. Can et al. (2004) found that female soccer players had significantly different body fat percentages $(19.7 \pm 0.7)$ and lean body mass from non-athletes, but there were no differences in overall absolute fat. Goalkeepers were the heaviest players with the highest percentage of body fat, but were the tallest players on the team (Ingebrigtsen et al., 2011; Milanovic et al., 2012). Contrastingly, forwards were the lightest with the lowest percentage of body fat (Ingebrigtsen et al., 2011; Milanovic et al., 2012).

Existing research considering morphological characteristics of female soccer players (Can et al., 2004; Ingebrigtsen et al., 2011; Milanovic et al., 2012) concluded that elite female soccer players were of average height (160 to $169 \mathrm{~cm}$ ) and weight (52 to $65 \mathrm{~kg}$ ), which did not differ from the unselected population of healthy women. Average body fat values ranged from 16 to $23 \%$ indicating mesomorph and endomorph characteristics.

Ingebrigtsen et al. (2011) found no significant differences in anthropometric variables between players grouped by a playing position. Although not significantly different, defenders were on average $5 \mathrm{~cm}$ taller than attackers and had higher body mass than the other positions. Krustrup et al. (2005) also found defenders to be taller and heavier than the other playing positions. They also found that midfielders had the lowest body fat percentage $(12.5 \pm 2.2)$. In contrast to physiological variables, there were no differences in body height, body mass and the percentage of body fat between top-level, high level and amateur female soccer players. 


\section{Physiological demands}

Soccer is characterized by intermittent activity ranging from walking to sprinting, thus valid information regarding activity patterns in women's soccer is essential for understanding the physical demands of the game. While there is a wealth of this type of information for male soccer, the same cannot be said for the women's game (Krustrup et al., 2005). Physiological demands are similar between men's and women's soccer, but competitive women's matches were characterized by less distance covered (nearly 33\% less), although at higher intensity levels (maximum speeds greater than $15 \mathrm{~km} / \mathrm{h}$ ) than typically found in the men's game (Krustrup et al., 2005). Timemotion studies in women's soccer revealed that most match play was spent on low-intensity activities i.e., standing, walking and jogging (Andersson et al., 2010; Krustrup et al., 2005; Mohr et al., 2008), where the level of competition influenced the total distance covered (Gabbett and Mulvey, 2008). The most common values for distances covered were in the range from 9000 to $11000 \mathrm{~m}$, depending on the competition level i.e., elite, sub-elite or amateur (Andersson et al., 2010; Gabbett and Mulvey, 2008; Krustrup et al., 2005; Mohr et al., 2008). Gabbett and Mulvey (2008) found that the total distance covered varied between $9324 \pm 840 \mathrm{~m}$ for a domestic match, 9706 $\pm 484 \mathrm{~m}$ in a national league match and $9968 \pm$ $1143 \mathrm{~m}$ in an international competition. They also reported that during international games players spent $15.7 \%$ of total game time standing, $50.1 \%$ walking, $26.7 \%$ jogging, $7.5 \%$ running at high intensity, $4.8 \%$ striding and $2.7 \%$ sprinting. Very similar results were found by Krustrup et al. (2005) who stated that female players spent $16 \%$ of time standing, $44 \%$ walking, $34 \%$ on low intensity activity and $4.8 \%$ running at high intensity. However, there was a wide range of movement patterns dependent on the player's position. Interestingly, they also reported that the distance covered at high intensity decreased $(p<0.05)$ from the first to the last $15 \mathrm{~min}$ period by $30 \%$ in the first half (270 to $190 \mathrm{~m})$ and $34 \%$ in the second half (240 to $160 \mathrm{~m}$ ). Krustrup et al. (2005) concluded that the mean value for total distance covered by female soccer players was $10300 \mathrm{~m}$, with high intensity running performed 125 times, for $2.3 \mathrm{~s}$ on average, covering $1310 \mathrm{~m}$; 26 sprints performed per game and approximately 1400 activity changes undertaken on average every 4 seconds.

Mohr et al. (2008) found that top class female soccer players covered less distance in the second half compared to the first (5 056 vs. $5286 \mathrm{~m}$ ), whereas no difference was observed for high level players (5 216 vs. $5226 \mathrm{~m}$ ). This may have been because, in this study, the top class players had covered less distance in high-intensity running and sprinting in the second compared to the first half, whereas the high level players only covered less distance when sprinting. Similarly, Andersson et al. (2010) found that top class players ran $28 \%$ longer at high intensity and sprinted $24 \%$ more than high level players. Also, the top class players performed approximately $40 \%$ less high intensity running in the last 15 minutes of a game compared to previous game times (Mohr et al., 2008). This may be due to players simply trying to maintain possession and run the clock down when they were in an unassailable position. In contrast, high level players performed less high intensity running at the end of both halves, considered to be critical periods of the game, and therefore suggestive of being due to fatigue i.e., disturbed muscle ion homeostasis, depletion of muscle glycogen and dehydration (Mohr et al., 2008).

No differences were found for the total distance covered between playing positions (Gabbett and Mulvey, 2008; Mohr et al., 2008), although defenders completed less high intensity running than midfielders and attackers, who covered the same distance. Attackers covered the longest distance when sprinting $(520 \mathrm{~m})$ compared to midfielders $(430 \mathrm{~m})$ and defenders $(330 \mathrm{~m})$. All playing positions completed less high intensity running and sprinting in the second compared to the first half. When analyzing different playing standards, midfielders covered more total distance, performed more high intensity runs and sprinted significantly longer (43\% more) in international matches compared to domestic games, whereas no such differences were found for defenders (Andersson et al., 2010; Gabbett and Mulvey, 2008).

\section{Physical abilities}

The most significant patterns of play found to determine the final score in soccer were: 1 vs. 1 play in defence and offence, accuracy of 
passes and effective execution of dead-ball situations (Soroka and Bergier, 2010). Consequently, optimal morphological characteristics and functional abilities beneficial to female soccer players should ideally include high levels of speed, agility, strength, power, endurance, accuracy, flexibility, balance and coordination. Specific conditioning programmes are used to improve each of these different components which can also include the development of motor abilities. Muscular strength, as well as anaerobic power, can be important for female soccer players to increase running performance through force production, intramuscular glycogen or increased anaerobic enzyme activity, improvements in the stretchshortening cycle or the force development rate (Sporiš et al., 2011). Strength and power training is used to increase force production for game situations requiring explosiveness (jumps, turns, kicks) to prevent injuries and decrease recovery time. Similarly, explosive strength training can be effective since it enhances neuronal activation and muscular adaptation (Sporiš et al., 2011).

The implementation of functional training focused on soccer-specific drills and plyometrics in order to improve explosive power may help conditioning in female soccer players as well as decrease the risk of anterior cruciate ligament injuries which are 3-8 times more likely to happen in female athletes (Bien, 2011; Grandstrand et al., 2006). Plyometric training includes jumps and hops in order to influence the stretch-shortening cycle of the muscle. Rubley et al. (2011) investigated the effectiveness of low-impact and low-frequency plyometric training in adolescent female soccer players and found that the plyometric group showed a significant increase in kicking distance from pre-test to week 7 (11.5\%) and an increase of $11.3 \%$ from week 7 to 14 . Furthermore, the control group showed a significant decrease in kicking distance between the pretest and week $14(15.6 \%)$ and a significant decrease between week 7 and 14 (11.6\%). The implementation of low-intensity and lowfrequency plyometric training into a regular training process once a week can increase lowerbody power (vertical jumping and kicking distance) if the frequency of training ( 1 day per week) allows adequate time for rest and recovery (Rubley et al., 2011).
Muscular strength and power in female soccer players can be improved by resistance training (Oberacker et al., 2012; Sporiš et al., 2011). This type of training, performed on unstable surfaces, improved balance, force production and power in female soccer players (Oberacker et al., 2012; Sporiš et al., 2011) as it induced neuromuscular stress and theoretically greater neural adaptations compared to resistance training on stable surface. However, no additional benefits for speed or explosive strength performance were found (Oberacker et al., 2012). Consequently, resistance training on unstable surfaces is not recommended for developing strength or agility (Oberacker et al., 2012), but should rather be considered a method to improve balance and decrease the risk of injuries.

Female players perform 1000 to 1500 movement changes, with approximately 700 direction changes at angles of up to 180 degrees, during a typical soccer match (Upton and Ross, 2011). Accordingly, agility is an essential attribute for successful performance, especially when accelerating, decelerating and reaccelerating (Yap and Brown, 2000). Usual sprint distances in soccer are between 5 and $10 \mathrm{~m}$ indicating that acceleration i.e., the rate of change in velocity, is more important than maximal velocity. Significant improvements in maximum velocity, measured by the 40 -yd sprint, were demonstrated as a consequence of both assisted and resisted sprint training compared to traditional training (Upton, 2011). Also, female soccer players increased their acceleration during the initial 5 yds $(4.6 \mathrm{~m})$ and $15 \mathrm{yds}(13.7 \mathrm{~m})$ after an assisted training program, whilst players in the resisted training group increased their acceleration during the final 25 yds (22.9 m) (Upton and Ross, 2011). Resisted sprint training also increased vertical jump height and peak power, although $13.7 \mathrm{~m}$ sprint and T-test time remained unchanged (Upton and Ross, 2011). Improvements in short distance speed and speed with direction changes, in the absence of an increase in peak power, indicated that neuromuscular factors combined with supramaximal training brought benefits in female soccer players. Resisted training provoked strength improvements while assisted training was linked to neurological adaptations as improvements were found for short distances (4.6 and $13.7 \mathrm{~m}$ ). These findings suggest that both 
assisted and resisted training improve $40 \mathrm{yd}$ maximal velocity (Upton and Ross, 2011; Upton, 2011). The improved acceleration for distances up to 5 and $15 \mathrm{yds}$ found for assisted training may be the most useful in female soccer due to similar game demands. Improved acceleration up to 25 yds $(22.9 \mathrm{~m})$ could be more beneficial for wingers, whose playing position demands longer distance runs.

Sprint performance in female soccer players can be improved using different methods of training such as resistance, speed and strength, velocity-specific strength and movement specific sprint associated exercises (Milanović et al., 2014). Assisted training enables a player to run faster than their maximal speed (over speed training) by increasing stride length, stride frequency, activity of the neuromuscular system as well as reducing flight time and ground reaction time (Milanović et al., 2014). Sprinting relies on the effective use of muscle stiffness during the stretch-shortening cycle (McCurdy et al., 2010). These researchers found a strong relationship between unilateral vertical jump performance and sprint performance which indicated that enhancement in this jump height would improve the flight time to concentric contact time ratio and thus potentially increase 25-m sprint performance in female soccer players. Coaches are therefore advised to consider integrating both unilateral drop and countermovement jumps into a training program in order to improve sprint performance. However, stride length, stride frequency and hand/arm action also have to be increased to improve speed (Yap and Brown, 2000).

\section{Female soccer players' injuries}

Of all soccer injuries, from 57 to $88 \%$ are related to lower extremities (Nyland et al., 1997; Rahnama et al., 2002). The most common lower extremity injuries in soccer are ankle sprains (16 to $36 \%$ ), followed by knee injuries (11.7 to $14 \%$; (Nyland et al., 1997). Furthermore, soccer is a sport with the highest rate of meniscal knee injuries. Injury risk is higher during the first and last 15 minutes of the match thought to be due to intense engagement at the beginning and fatigue towards the end of the two halves (Rahnama et al., 2002). Furthermore, injury risk is higher in the attacking and defending zones where ball possession is most vigorously contested.
Fifty percent of injuries are sprains for female soccer players who are also up to three times more likely to suffer traumatic knee injuries (ligaments) than males (Nyland et al., 1997). Jacobson and Tegner (2007) recorded injury occurrences in 269 elite female soccer players in Sweden during one year finding $82 \%$ lower extremity injuries and $28 \%$ of these were reinjuries. Twenty two percent were major injuries, mostly to the knee. Söderman et al. (2001) reported that $41 \%$ of female soccer players sustained injuries during one season; $66 \%$ of them traumatic and most occurred during the match. Eighty nine percent were related to lower extremities with $42 \%$ of them being ankle or knee injuries. The most frequent injuries were ankle sprains and the most traumatic ones were knee ligament injuries.

\section{Conclusions}

According to the studies reviewed, coaches should individualize training depending on players' physical characteristics while ensuring that all players are able to perform at the match intensity levels associated with their playing position. Female soccer coaches should seek to improve players' abilities to perform repeated exercise at high intensity to replicate the stop and go nature of soccer. Both aerobic and anaerobic energy systems are well utilized during matches and training needs to increase players' ability to perform high intensity exercise and improve their ability to recover between these activities. While high speed motion only contributes to about $11 \%$ of the total distance covered, high intensity actions are likely to be performed at the most important moments of the game e.g., competing for ball possession or contributing to scoring or conceding a goal. It seems logical, therefore, that female players with better functional abilities are able to perform more sprints, play longer at high intensity and have shorter periods of recovery. This would explain the positive correlations between $\mathrm{VO}_{2 \max }$, distance covered and sprint performance. However, simple information regarding work rates, in the absence of match analysis information, cannot determine the extent to which simply sprinting more often and for longer would improve female soccer performance 


\section{References}

Andersson HÅ, Randers MB, Heiner-Møller A, Krustrup P, Mohr M. Elite female soccer players perform more high-intensity running when playing in international games compared with domestic league games. J Strength Cond Res, 2010; 24(4): 912-919

Bangsbo J. The physiology of soccer--with special reference to intense intermittent exercise. Acta Physiol Scand Suppl, 1993; 619: 1-155

Bien DP. Rationale and implementation of anterior cruciate ligament injury prevention warm-up programs in female athletes. J Strength Cond Res, 2011; 25(1): 271-285

Can F, Yilmaz I, Erden Z. Morphological characteristics and performance variables of women soccer players. J Strength Cond Res, 2004; 18(3): 480-485

Davis JA, Brewer J. Applied physiology of female soccer players. Sports Med, 1993; 16(3): 180-189

Gabbett TJ, Mulvey MJ. Time-motion analysis of small-sided training games and competition in elite women soccer players. J Strength Cond Res, 2008; 22(2): 543-552

Grandstrand SL, Pfeiffer RP, Sabick MB, DeBeliso M, Shea KG. The effects of a commercially available warm-up program on landing mechanics in female youth soccer players. J Strength Cond Res, 2006; 20(2): 331-335

Grieco CR, Cortes N, Greska EK, Lucci S, Onate JA. Effects of a combined resistance-plyometric training program on muscular strength, running economy, and Vo2peak in division I female soccer players. J Srength Cond Res, 2012; 26(9): 2570-2576. doi: 10.1519/JSC.0b013e31823db1cf

Ingebrigtsen J, Dillern T, Shalfawi SA. Aerobic capacities and anthropometric characteristics of elite female soccer players. J Strength Cond Res, 2011; 25(12): 3352-3357

Jacobson I, Tegner Y. Injuries among Swedish female elite football players: a prospective population study. Scand J Med Sci Sports, 2007; 17(1): 84-91

Krustrup P, Mohr M, Ellingsgaard H, Bangsbo J. Physical demands during an elite female soccer game: importance of training status. Med Sci Sports Exerc, 2005; 37(7): 1242-1248

Martens R. Successful Coaching: Human Kinetics; 1996

Matković BR, Mišigoj-Duraković M, Matković B, Janković S, Ružić L, Leko G, Kondrič M. Morphological differences of elite Croatian soccer players according to the team position. Coll Antropol, 2003; 27(1): $167-174$

McCurdy KW, Walker JL, Langford GA, Kutz MR, Guerrero JM, McMillan J. The relationship between kinematic determinants of jump and sprint performance in division I women soccer players. J Strength Cond Res, 2010; 24(12): 3200-3208

Milanovic Z, Sporis G, Trajkovic N. Differences in body composite and physical match performance in female soccer players according to team position. Journal of Human Sport and Exercises, 2012; 7(1): S67S72

Milanović Z, Sporiš G, James N, Trajković N. Motor Abilities of Female Soccer Players. Sport Science and Disability, 2014: 22

Mohr M, Krustrup P, Andersson H, Kirkendal D, Bangsbo J. Match activities of elite women soccer players at different performance levels. J Strength Cond Res, 2008; 22(2): 341-349

Nyland JA, Caborn DN, Brosky JA, Kneller CL, Freidhoff G. Anthropometric, muscular fitness, and injury history comparisons by gender of youth soccer teams. J Strength Cond Res, 1997; 11(2): 92-97

Oberacker L, Davis S, Haff G, Witmer C, Moir G. The effects of stable and unstable surface resistance training on performance of female soccer players. J Srength Cond Res, 2012; 26(10): 2734-2740

Olson JR. VO2 peak and running economy in female collegiate soccer players across a competitive season. Master Master of Science (M.S.) in Exercise and Sport Science, Oregon State University; 2008

Polman R, Walsh D, Bloomfield J, Nesti M. Effective conditioning of female soccer players. J Sports Sci, 2004; 
22(2): 191-203

Rahnama N, Reilly T, Lees A. Injury risk associated with playing actions during competitive soccer. Brit J Sport Med, 2002; 36(5): 354-359

Reilly T, Secher N, Snell P, Williams C. Physiology of sports: An overview. Physiology of sports, 1990: 465-485

Rey E, Lago-Peñas C, Lago-Ballesteros J. Tensiomyography of selected lower-limb muscles in professional soccer players. J Electromyogr Kinesiol, 2012; 22(6): 866-872

Rubley MD, Haase AC, Holcomb WR, Girouard TJ, Tandy RD. The effect of plyometric training on power and kicking distance in female adolescent soccer players. J Strength Cond Res, 2011; 25(1): 129-134

Söderman K, Adolphson J, Lorentzon R, Alfredson H. Injuries in adolescent female players in European football: a prospective study over one outdoor soccer season. Scand J Med Sci Sports, 2001; 11(5): 299304

Soroka A, Bergier J. Actions with the Ball that Determine the Effectiveness of Play in Women's Football. Journal of Human Kinetics, 2010; 26: 97-104

Sporiš G, Jovanović M, Krakan I, Fiorentini F. Effects of strength training on aerobic and anaerobic power in female soccer players. Sport Science, 2011; 4(2): 32-37

Upton D, Ross J. Assisted and Resisted Sprint Training: Effects on 13.7 M Speed, Speed with Direction Change, and Peak Power in Division I Female Soccer Athletes. J Strength Cond Res, 2011; 25: S83-S84

Upton DE. The effect of assisted and resisted sprint training on acceleration and velocity in Division IA female soccer athletes. J Strength Cond Res, 2011; 25(10): 2645-2652

Yap CW, Brown LE. Development of speed, agility, and quickness for the female soccer athlete. Strength $\mathcal{E}$ Conditioning Journal, 2000; 22(1): 9

\section{Corresponding author:}

\section{Zoran Milanović, PhD}

Faculty of Sport and Physical Education

Čarnojevićeva 10a

18000 Niš

Phone: 00381637399366

E-mail: zoooro_85@yahoo.com 